\title{
Overexpression of the serpin megsin induces progressive mesangial cell proliferation and expansion
}

\author{
Toshio Miyata, ${ }^{1}$ Reiko Inagi, ${ }^{1}$ Masaomi Nangaku, ${ }^{2}$ Toshiyuki Imasawa, ${ }^{1}$ Masahiro Sato, ${ }^{1}$ \\ Yuko Izuhara, ${ }^{1}$ Daisuke Suzuki, ${ }^{1}$ Atsusi Yoshino, ${ }^{1}$ Hiroshi Onogi, ${ }^{1}$ Minoru Kimura, ${ }^{1}$ \\ Satoshi Sugiyama, ${ }^{3}$ and Kiyoshi Kurokawa ${ }^{1}$
}

${ }^{1}$ Molecular and Cellular Nephrology, Institute of Medical Sciences and Department of Internal Medicine,
Tokai University School of Medicine, Kanagawa, Japan
${ }^{2}$ Division of Nephrology and Endocrinology, University of Tokyo School of Medicine, Tokyo, Japan
${ }^{3}$ Division of Nephrology, Fujita Health University School of Medicine, Aichi, Japan

Address correspondence to: Toshio Miyata, Institute of Medical Sciences and Department of Medicine, Tokai University School of Medicine, Bohseidai, Isehara, Kanagawa 259-1193, Japan.

Phone: 81-463-93-1936; Fax: 81-463-93-1938; E-mail: t-miyata@is.icc.u-tokai.ac.jp.

Received for publication October 3, 2001, and accepted in revised form January 28, 2002.

\begin{abstract}
Mesangial cells maintain normal glomerular function by mediating ECM remodeling and immune complex disposal. We have recently identified megsin, a novel member of the serine protease inhibitor (serpin) superfamily predominantly expressed in the mesangium. While our previous studies suggested a role for megsin in the pathogenesis of human glomerular diseases, its exact biological significance remained unknown. Here we produced two lines of megsin transgenic mice. Overexpression of megsin led to progressive mesangial matrix expansion and an increase in the number of mesangial cells. These glomerular lesions were accompanied by an augmented immune complex deposition, together with Ig's and complement. Binding and functional assays in vitro identified plasmin as one biological substrate of megsin and confirmed its activity as a proteinase inhibitor. Transgenic animals exhibiting nephritis as a result of treatment with anti-glomerular basement membrane antiserum showed significantly more persistent expansion of the mesangial ECM than was seen in parental mice. Megsin therefore exerts a biologically relevant influence on mesangial function, and on the mesangial microenvironment, such that simple overexpression of this endogenous serpin engenders elementary mesangial lesions.
\end{abstract}

J. Clin. Invest. 109:585-593 (2002). DOI:10.1172/JCI200214336.

\section{Introduction}

Mesangial cells play a central role in maintaining both structure and function of the glomerulus. In order to elucidate pathogenesis of glomerular diseases, we recently cloned a new human mesangium-predominant gene, megsin, which is a new member of the serine protease inhibitor (serpin) superfamily (1). The amino acid sequence in the reactive loop site of megsin exhibits the characteristic features of functional serpins. Northern blot and RT-PCR analyses of various tissues and cells demonstrated that megsin was predominantly expressed in human mesangial cells. These findings were further confirmed by in situ hybridization $(1,2)$ and by immunohistochemistry using megsin-specific antibodies (3). In IgA nephropathy and diabetic nephropathy, megsin mRNA expression in glomeruli was upregulated $(1,2)$. A similar upregulation of megsin was observed in the experimental antiThy1 nephritis model of rats (4).

To further understand a role of megsin in mesangial function, we overexpressed the human megsin cDNA in the mouse genome. Two lines of megsin transgenic mice have been obtained. They developed progressive mesangial matrix expansion, an increase in the number of mesangial cells, and an augmented immune complex deposition. Our in vitro assays utilizing recombinant megsin confirmed that megsin serves as a functional serpin. These findings demonstrate that megsin exerts a biologically relevant influence on mesangial function.

\section{Methods}

Megsin transgenic mice. To generate the human megsin transgene construct, the entire coding sequence of megsin cDNA was subcloned in the sense orientation into the pBsCAG-2 (5). The megsin transgene isolated by digestion of pBsCAG-2 containing megsin cDNA was microinjected into one pronucleus of fertilized B6C3F $1 \times \mathrm{C} 57 \mathrm{BL} / 6 \mathrm{~N}$ hybrid eggs, followed by transfer into the oviducts of pseudopregnant mice as described elsewhere (6). Mouse genomic DNA extracted from tail tissue was used to detect the transgene by Southern blot analysis with megsin transgene probe. Simultaneously, transgenic mice were also identified by PCR using specific primers for megsin or $\mathrm{PBsCAG}-2$ vector. Primers for the cytomegalovirus enhancer $(\operatorname{Pr} 1$ in Figure 1a) were CMV-F1 (5'-GTC GAC ATT GAT TAT TGA CTA G-3') and CMV-R1 (5'-CCA TAA GGT CAT GTA CTG$3^{\prime}$ ), with an amplified 250-bp fragment. Primers for the 
$5^{\prime}$ junction between vector and inserted megsin gene (Pr2) were $\beta$-gl-3 ( $5^{\prime}$-CTT CTG GCG TGT GAC CGG CG- $3^{\prime}$ ) and hM2-2 (5'-TCA CAA TGC TGA GAT CAT AAT CCT TGT GGG ATG C-3'), with an amplified 400-bp fragment. Primers for the $3^{\prime}$ junction between vector and inserted megsin gene (Pr3) were hM8-1 (5'-TTA TTC AGT GGC AAA GTT TCT TGC CCT TGA- $3^{\prime}$ ) and $\beta$-globin R ( $5^{\prime}$-TCG AGG GAT CTT CAT AAG AGA AGA G-3'), with an amplified 563-bp fragment.

Animals were treated in accordance with the guidelines of the Committee on Ethical Animal Care and Use of Tokai University. Urine was collected 1 day before sacrifice by cervical dislocation. Urinary albumin excretion was measured by a kit (Mouse Albumin ELISA Quantitation Kit; Bethyl Laboratories, Montgomery, Texas, USA) according to the manufacturer's protocol. Blood samples were also obtained at the time of sacrifice $(6,15,20$, and 40 weeks) for hematological and biochemical analyses.

ELISA for circulating immune complexes. Levels of circulating immune complexes were determined by the $\mathrm{C} 1 \mathrm{q}$ ELISA as previously reported by Sekine et al., with some modifications (7). Briefly, microtiter wells coated with human C1q (Sigma Chemical Co., St. Louis, Missouri, USA) were incubated with serum samples diluted 1:50 with PBS containing 1\% BSA, $0.01 \mathrm{M}$ EDTA, and $0.05 \%$ Tween- 20 overnight at $4{ }^{\circ} \mathrm{C}$. After washing with PBS containing $0.05 \%$ Tween- 20 , the plate was reacted with peroxidase-conjugated goat anti-mouse IgG ( $\gamma$-chain-specific; Sigma Chemical Co.) for 3 hours at room temperature, followed by reaction with substrate solution containing $o$-phenylenediamine dihydrochloride and $0.012 \% \mathrm{H}_{2} \mathrm{O}_{2}$ for color development. Then the absorbance at $492 \mathrm{~nm}$ was measured. Each sample was measured in duplicate. The sera of 25 -week-old MRL/lpr mice (7) were used as a positive control.

Northern blot analysis. Total RNA was isolated from the quickly frozen kidney tissues using ISOGEN (Wako Pure Industries Ltd., Osaka, Japan). Twenty micrograms of RNA was electrophoresed on a $1 \%$ agarose-formaldehyde gel, followed by capillary transfer and hybridization using a human megsin cDNA fragment as a probe.

Western blot analysis. Kidney tissues $(10 \mathrm{mg})$ were homogenized in $100 \mu \mathrm{l}$ of $0.35 \mathrm{M}$ Tris- $\mathrm{HCl}(\mathrm{pH}$ 6.8) containing $10 \%$ SDS, $36 \%$ glycerol, $5 \% \beta$-mercaptoethanol, and $0.012 \%$ bromophenol blue and centrifuged at 5,000 $\mathrm{g}$ for 15 minutes. Megsin protein was detected by rabbit anti-human megsin IgG $(10 \mu \mathrm{g} / \mathrm{ml})$ (3) as the first antibody and alkaline phosphatase-conjugated goat anti-rabbit IgG (ICN Pharmaceuticals Inc.-Cappel Research Products, Costa Mesa, California, USA) as the secondary antibody. As a positive control, fusion megsin protein with maltose-binding protein expressed in Escherichia coli was used (3).

Histopathology. Kidneys were fixed in $4 \%$ neutral buffered formaldehyde, embedded in paraffin, and sectioned at $4 \mu \mathrm{m}$ thickness, followed by the periodic acid-Schiff (PAS) staining for light microscopic analysis and morphometry.

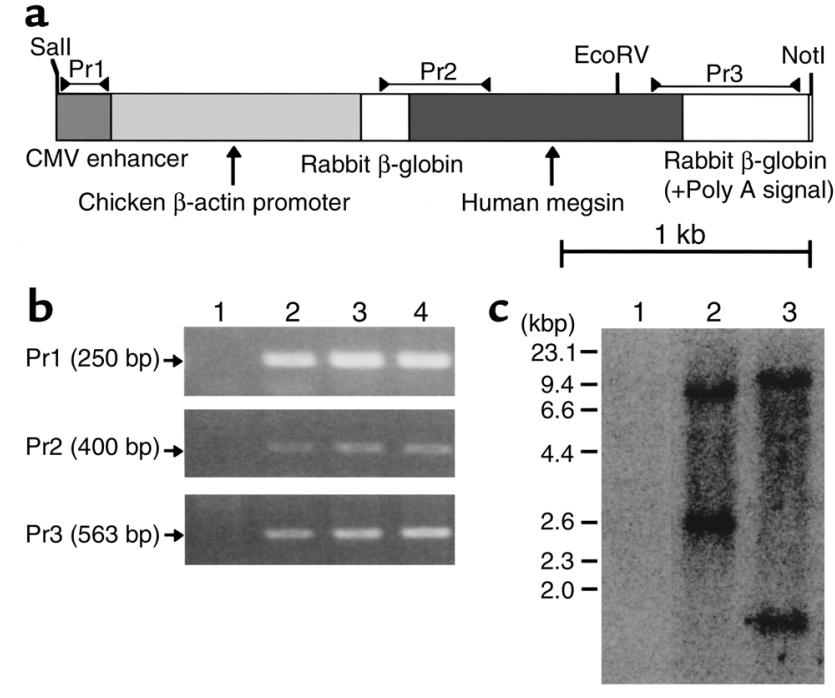

\section{Figure 1}

Generation and characterization of human megsin transgenic mice. (a) Megsin transgene construct. Full-length human megsin cDNA was subcloned in the rabbit $\beta$-globin gene including a part of the second intron, the third exon, and the $3^{\prime}$ untranslated region. The positions of primers for PCR analysis are indicated above the construct. (b) Identification of human megsin transgene by PCR of genomic DNA. Lane 1, a wild-type mouse DNA; lane 2, a wild-type mouse DNA with one copy of megsin transgene added; lane $3, F_{0}$ megsin transgenic DNA (line A); lane 4, $F_{0}$ megsin transgenic DNA (line B). (c) Identification of human megsin transgene by genomic Southern blot analysis. Southern blot analysis after EcoRV digestion of genomic DNA. Lane 1, a wild-type mouse DNA; lane 2, $F_{0}$ megsin transgenic DNA (line A); lane 3, $F_{0}$ megsin transgenic DNA (line B). Approximately $9.0 \mathrm{~kb}$ and $2.6 \mathrm{~kb}$ of fragments in line $A$ and $10.0 \mathrm{~kb}$ and 1.5 $\mathrm{kb}$ of fragments in line $\mathrm{B}$, but not endogenous murine megsin genome, are detected with human megsin transgene probe.

Immunobistochemistry. In order to examine ECM deposition, 4- $\mu \mathrm{m}$ sections of methyl Carnoy's fixed or frozen tissues were stained with antibodies as follows. Type IV and type I collagen was localized using an indirect immunoperoxidase method with a polyclonal goat anti-type IV collagen and anti-type I collagen antibody, respectively (Southern Biotechnology Associates, Birmingham, Alabama, USA). Fibronectin was identified using the rabbit anti-fibronectin antibody (Chemicon International, Temecula, California, USA). Laminin was identified using the rabbit anti-laminin antibody (Chemicon International). For detection of human megsin products, frozen sections were incubated with rabbit anti-human megsin $\operatorname{IgG}(10 \mu \mathrm{g} / \mathrm{ml})$ as the first antibody, followed by peroxidase-conjugated swine anti-rabbit antibody (DAKO A/S, Glostrup, Denmark) as the secondary antibody. The developed tissues were counterstained with hematoxylin.

Immunofluorescence. Frozen sections were incubated with FITC-conjugated goat anti-mouse IgG, IgA, IgM, or C3 antibodies (Cappel Research Products). All tissues were cut, stained, and analyzed in a single setting to minimize effects due to variations in tissue processing or in the intensity of the ultraviolet light source. In order to 
detect leukocyte infiltration, mAb's against mouse macrophage F4/80 antigen, neutrophil antigen, CD4 antigen, and CD8a antigen were purchased from Caltag Laboratories Inc. (Burlingame, California, USA). A semiquantitative scoring system was used for evaluation of mouse IgG deposition as follows: grade 1, normal; grade $2,0-25 \%$ of glomerular area involved; grade $3,25-50 \%$; grade 4, 50-75\%; and grade 5, >75\%. Glomerular cross sections containing only a minor portion of the glomerular tuft (<20 discrete capillary segments per cross section) were not used. The glomeruli were scored in more than 20 glomeruli cross sections per specimen in a blinded manner by two pathologists independently. Electron microscopy. Tissue samples were immersed for 2 hours in $0.1 \mathrm{M}$ sodium phosphate buffer ( $\mathrm{pH} 7.4$ ) containing $2 \%$ glutaraldehyde, postfixed in $2 \%$ osmium tetroxide, and embedded in Epon 812 (TAAB Laboratories Equipment Ltd., Berkshire, United Kingdom). Ultrathin sections were stained with uranyl acetate and lead aceton and analyzed by electron microscopy (JEM1200EX; JEOL Ltd., Tokyo, Japan).

Morphometry. PAS-stained kidney sections described above were used for morphometric analysis. Each staining picture was scanned using a 3CCD camera (Olympus Optical Co., Tokyo, Japan), and the glomerular tuft areas, glomerular sizes, and numbers of glomerular cell nuclei were analyzed in a blinded manner using the software Image Grabber PCI (Fuji Photo Film Co., Tokyo, Japan) and Mac Aspect (Mitani Co., Tokyo, Japan). The glomerular size was defined by encircling the outer region of the glomerular capillary tuft. Twenty consecutive glomeruli in the midcortex were measured. Glomerular cross sections containing only a minor portion of the glomerular tuft ( $<20$ discrete capillary segments per cross section) were not used. In order to avoid examiner's bias, both the largest and the smallest glomerulus in the 20 glomeruli were excluded. Eighteen glomeruli from each section were examined, and averages were expressed as means $\pm \mathrm{SD}$.

Anti-glomerular basement membrane (GBM) nephritis. The preparation of rabbit anti-glomerular basement membrane (anti-GBM) antiserum was performed as described by Hisada et al. (8) with some modifications. Anti-GBM nephritis was induced in either megsin transgenic mice (line A) or wild-type littermates at 8 weeks of age. The mice were immunized intraperitoneally with $0.025 \mathrm{mg} / \mathrm{g}$ body weight of rabbit IgG (Organon Teknika Corp., West Chester, Pennsylvania, USA) emulsified with CFA. Five days after immunization, $100 \mu \mathrm{l}$ of anti-GBM antiserum was injected intravenously via tail vein. In preliminary experiments, we confirmed that $100 \mu \mathrm{l}$ of the anti-GBM antiserum induced glomerulonephritis with mesangial expansion with complete recovery of the manifestations by 4 months after the induction.

At 6 hours $(n=5), 3$ days $(n=5), 7$ days $(n=5)$, and 28 days $(n=10)$ after administration of anti-GBM antiserum to both megsin transgenic and wild-type mice, the mice were sacrificed. The obtained kidneys were stained by periodic acid-methenamine-silver and subsequently assessed for the mesangial matrix expansion by scoring 1 to 4 : grade 1, normal; grade 2, mild (less than one-third of glomerular tuft area); grade 3 , moderate (less than two-thirds of glomerular tuft area); and grade 4, severe (greater than two-thirds of glomerular tuft area). The glomeruli were scored in more than 20 glomeruli cross sections per specimen in a blinded manner by two pathologists independently.

Recombinant megsin. Recombinant human megsin was purified from the cultured supernatant of megsintransfected Chinese hamster ovary (CHO) cells (3). In brief, the entire coding sequence of megsin cDNA was tagged with c-myc and (His) 6 at the $\mathrm{N}$-terminus using PCR-based mutagenesis. The tagged megsin was cloned into pREP9 (Invitrogen Corporation, Carlsbad, California, USA), a mammalian expression vector. The stable megsin transfectants of $\mathrm{CHO}$ cells were obtained, and the megsin recombinant protein tagged with c-myc-(His) 6 (CHO-megsin) was purified from the culture supernatant by using the (His) ${ }_{6}$ affinity column.

Functional assays. Serine proteases including plasmin, kallikrein, elastase, trypsin, urokinase, cathepsin $\mathrm{G}$ (Sigma Chemical Co.), tissue plasminogen activator (t-PA; Biopool AB, Ventura, California, USA), and thrombin (Calbiochem-Novabiochem Corp., La Jolla, California, USA) were used. To examine complex formation of megsin with serine proteases, the purified megsin was incubated at $37^{\circ} \mathrm{C}$ for 30 minutes with each serine protease at 2:1 molar ratio in PBS ( $\mathrm{pH}$ 7.4) and analyzed by SDS-PAGE under a nonreducing condition, followed by Coomassie brilliant blue (CBB) staining or by immunoblot analysis (for t-PA) using sheep anti-human t-PA antibody (Cedarlane Laboratories Ltd., Hornby, Ontario, Canada).

The reaction mixture was also applied onto an aliphatic reverse phase chip (H4 Protein Chip; Ciphergen Biosystems Inc., Fremont, California, USA) and airdried. The chip was reacted with sinapinic acid, followed by mass analysis with surface-enhanced laser desorption/ionization time-of-flight (SELDI-TOF) mass spectrometry (SELDI Protein Biology System II; Ciphergen Biosystems Inc.).

To assess the inhibitory activity of megsin with plasmin activity, the purified megsin was incubated at $37^{\circ} \mathrm{C}$ for 30 minutes with plasmin at various molar ratios in $0.2 \mathrm{M}$ Tris- $\mathrm{HCl}$ ( $\mathrm{pH}$ 8.0). Each reaction mixture was further incubated with $1 \mathrm{mM}$ synthetic fluorometric plasmin substrate, $t$-butyloxycarbonyl-Glu-Lys-Lys-4-methylcoumaryl-7-amid (Boc-Glu-Lys-Lys-MCA) (Peptide Institute Inc., Osaka, Japan), followed by fluorometrical determination of the cleavage of peptide-MCA to amino4-methyl-coumarin (AMC) at the wavelength of excitation $(\lambda$ ex $)=380 \mathrm{~nm}$ and the wavelength of emission $(\lambda \mathrm{em})=460 \mathrm{~nm}$. Antiplasmin (Sigma Chemical Co.) was used as a control. In some experiments, the megsin activity was inactivated by incubation of purified megsin at $60^{\circ} \mathrm{C}$ for 15 hours in $0.25 \mathrm{M}$ trisodium citrate and 10 $\mathrm{mM}$ Tris- $\mathrm{HCl}(\mathrm{pH}$ 7.4). 


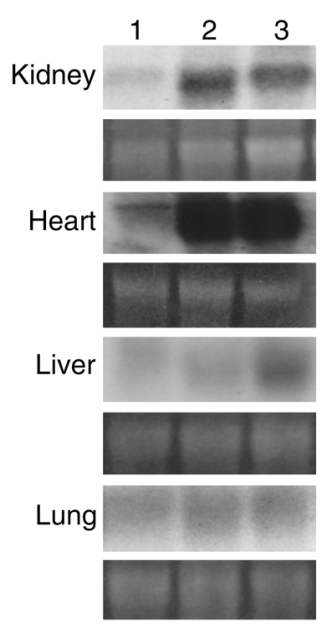

Figure 2

Northern blot analysis of megsin transgene. Upper panels: total RNAs of kidneys, hearts, livers, or lungs from a wild-type mouse (lane 1 ), an $F_{1}$ megsin transgenic mouse (line $A$ ) (lane 2), and an $F_{1}$ megsin transgenic mouse (line B) (lane 3). Lower panels: the ethidium bromide staining pattern of $18 \mathrm{~S}$ rRNA in each total RNA sample.

Statistical analysis. Data were expressed as means \pm SD. ANOVA was used to evaluate the statistical significance of various differences. If a significant difference was indicated by the analysis, Scheffe's $t$ test was used to compare results obtained from transgenic and nontransgenic control mice.

\section{Results}

Generation of megsin transgenic mice. To generate human megsin transgenic mice, the full-length megsin cDNA was overexpressed under control of the cytomegalovirus enhancer/chicken $\beta$-actin promoter system (Figure 1a). Of 56 putative founders, six had megsin transgene detected by PCR analysis of the genomic DNA. Two of the six transgenic founders were successfully bred to C57BL/6N mice, and two independent lines (lines A and B) were established. Both lines had 250-bp ( $\operatorname{Pr} 1$ in Figure 1), 400-bp ( $\operatorname{Pr} 2)$, and 563-bp (Pr3) fragments of megsin transgene by PCR analysis (Figure 1b). By Southern blot analysis of mouse genomic DNA, each lineage had a distinct integration pattern (Figure 1c). Southern blot and PCR analyses confirmed that both lines carried one copy of the transgene in their genomes.

Both lines transmitted the transgene to their progeny, and litter sizes were comparable to controls, suggesting normal reproductive behavior. Hematological and biochemical analyses of blood samples of transgenic $(n=12)$ and wild-type $(n=10)$ mice demonstrated no statistically significant difference between the two groups in the number of erythrocytes, white blood cells, or platelets. The serum levels of glucose, blood urea nitrogen, creatinine, cholesterol, transaminases, IgG, IgA, and IgM were also identical at 40 weeks after birth. The amounts of albuminuria did not differ between the wild-type and transgenic animals $(21.56 \pm 18.51 \mu \mathrm{g} / \mathrm{ml}$ vs. $23.28 \pm 11.17 \mu \mathrm{g} / \mathrm{ml})$. The levels of circulating immune complexes, measured by ELISA using $\mathrm{C} 1 \mathrm{q}$ binding assay, were significantly higher $(P<0.01)$ in transgenic mice $\left(\mathrm{OD}_{492}\right.$ $0.029 \pm 0.003)$ than in wild-type mice $\left(\mathrm{OD}_{492}\right.$ $0.018 \pm 0.001)$. As compared with the levels of circulating immune complexes in $\mathrm{MRL} / \mathrm{lpr}$ mice $\left(\mathrm{OD}_{492}\right.$ $0.215 \pm 0.014)$, these values were significantly lower $(P<0.001)$. Urine examination revealed no statistically significant difference between the two groups in the levels of urinary protein excretion.

While transgenic mice were grossly indistinguishable from their normal littermates at birth, transgenic progeny of the two lines subsequently developed renal disorders as described below.

Expression of human megsin. Northern blot analysis demonstrated successful overexpression of human megsin mRNA in the transgenic mice (Figure 2). The transgene was expressed in a variety of tissues. The expression levels of the transgene were relatively high in the kidney and the heart, while they were lower in the liver and were barely detectable in the lung. The

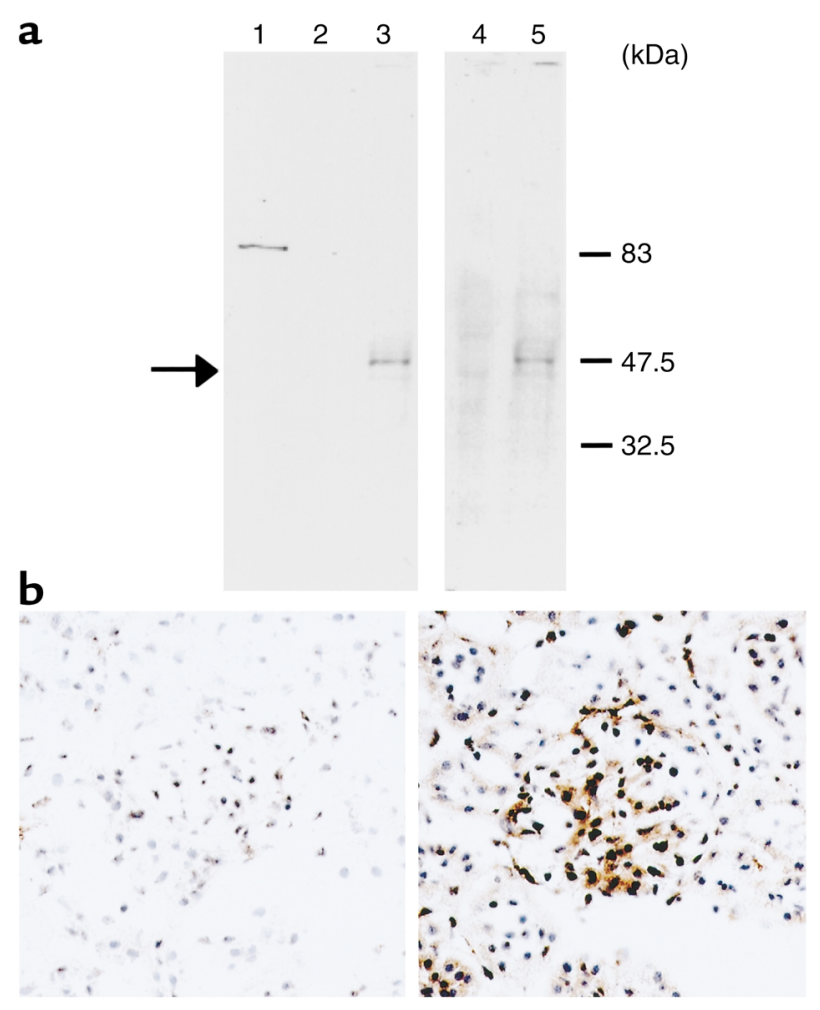

Figure 3

Western blot analysis of megsin gene product. (a) Characterization of anti-human megsin antibody. Lane 1, human megsin fusion protein with MBP expressed in E. coli ( $86 \mathrm{kDa})$; lanes 2 and 4, kidneys from wild-type mice; lane 3 , kidney from $F_{1}$ megsin transgenic mouse (line A); lane 5, kidney from $F_{1}$ megsin transgenic mouse (line B). Arrow indicates megsin product with an approximate molecular weight of $47 \mathrm{kDa}$. (b) Immunohistochemical detection of human megsin gene product in the kidneys of a wild-type mouse (left) and an $\mathrm{F}_{1}$ megsin transgenic mouse (line A) (right). $\times 100$. 


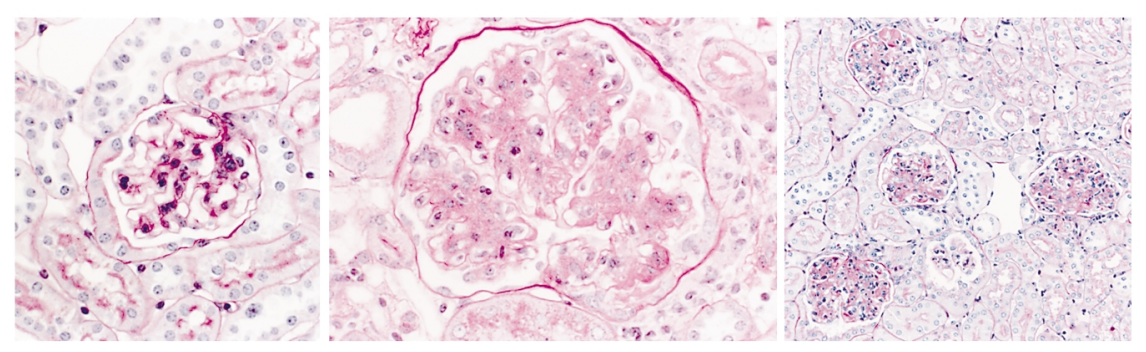

\section{Figure 4}

Histopathological analysis of murine kidneys. PAS staining. As compared with $40-$ week-old wild-type mice (left, $\times 200), F_{1}$ megsin transgenic mice (line $A$ ) of the same age developed mesangial matrix expansion and an increase in the number of mesangial cells (middle, $\times 200$; right, $\times 50$ ).

expression of human megsin cDNA in the transgenic kidney started at preimplantation stage and continued even after 40 weeks of age.

The protein expression of human megsin gene products in transgenic mice was confirmed by immunoblot analysis of kidney crude homogenates using antihuman megsin peptide antibody (Figure 3a). Immunohistochemical studies revealed the megsin protein expression ubiquitously in both glomeruli and tubules of these mice (Figure 3b). Their immunoreactivities were completely abolished after preincubation of the antibodies with an excess of human megsin peptide.

Transgenic mice developed mesangial matrix expansion and an increase in the number of mesangial cells. Until 15 weeks of age, megsin transgenic mice did not show any significant pathological changes. At 20 weeks, some mice from line A exhibited glomerular morphological changes, which became even more apparent at 40 weeks. These changes included an increased number of cells in the mesangial area and an expansion of mesangial matrix with deposits therein (Figure 4). Although megsin transgene was also expressed in the other tissues as described above, pathological changes detected histologically by light microscopy were observed exclusively in the kidney.

In order to quantify these glomerular changes, we performed computer-assisted morphometrical analyses. We measured glomerular sizes and numbers of mesangial cells in 25 wild-type mice and decided normal limits as a range of mean $+2 \mathrm{SD}$. Using this definition, 3 out of 14 heterozygous $F_{1}$ transgenic mice from line A developed either of these changes at 20 weeks after birth. At 40 weeks, among 19 heterozygous transgenic mice from line A, 11 (57.9\%) developed an increase in glomerular size and 12 (63.2\%) developed an increase in glomerular cell numbers (Figure 5). Altogether, our histological analysis revealed glomerular abnormalities in 14 (73.0\%) of 19 transgenic mice. In line B at 40 weeks, essentially the same glomerular abnormalities as in line A were observed in $18.2 \%$ of the transgenic animals (4 of 22). We did not observe any sex differences in either severity or penetrance of the disease. We did not detect any pathological changes in other organs. Except for one wild-type mouse, which developed mild expansion of mesangial matrix at 40 weeks, no wild-type mice exhibited pathological changes. Detailed analyses of glomerular abnormalities were performed as below by employing line $\mathrm{A}$ as representative animals.
Immunohistochemical analysis revealed accumulation of type IV collagen and laminin. In order to analyze abnormal glomeruli in the transgenic animals in detail, we performed immunohistochemical analysis of various ECM components (Figure 6). We did not observe accumulation of type I collagen in either wild-type or transgenic mice. Transgenic mice showed marked accumulation of type IV collagen and laminin in their sclerotic glomeruli. The amount of fibronectin was less in the sclerotic glomeruli of the transgenic animals than in those of wild-type mice.

Our immunohistochemical analysis and PAS staining revealed no infiltration of leukocytes at any time point, either in the glomerulus or in the tubulointerstitium. Therefore, it is likely that hypercellularity in the mesangial area of the transgenic mice was due to proliferation of resident glomerular cells.

Glomerular change was associated with an augmented immune complex deposition. Immunofluorescent studies were negative before 20 weeks of age. At 20 weeks we detected Ig's (IgA, IgG, and $\operatorname{IgM}$ ) and complement deposition in mouse glomeruli for the first time. Immune complex deposition increased in an age-dependent
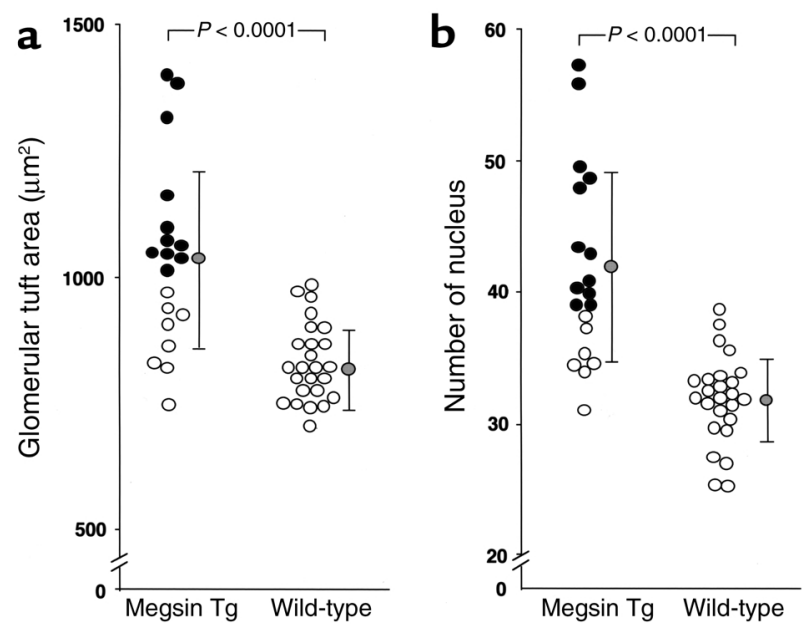

\section{Figure 5}

Computer-assisted morphometry of glomerular abnormalities in megsin transgenic mice. Glomerular tuft areas (a) and numbers of mesangial cells (b) were measured in 25 wild-type mice and 19 megsin transgenic mice (line A). Normal limits are considered as a range of mean +2 SD in wild-type mice. Filled circles represent animals above the normal limits, while open circles demonstrate mice within normal limits. The mean \pm SD is indicated. 


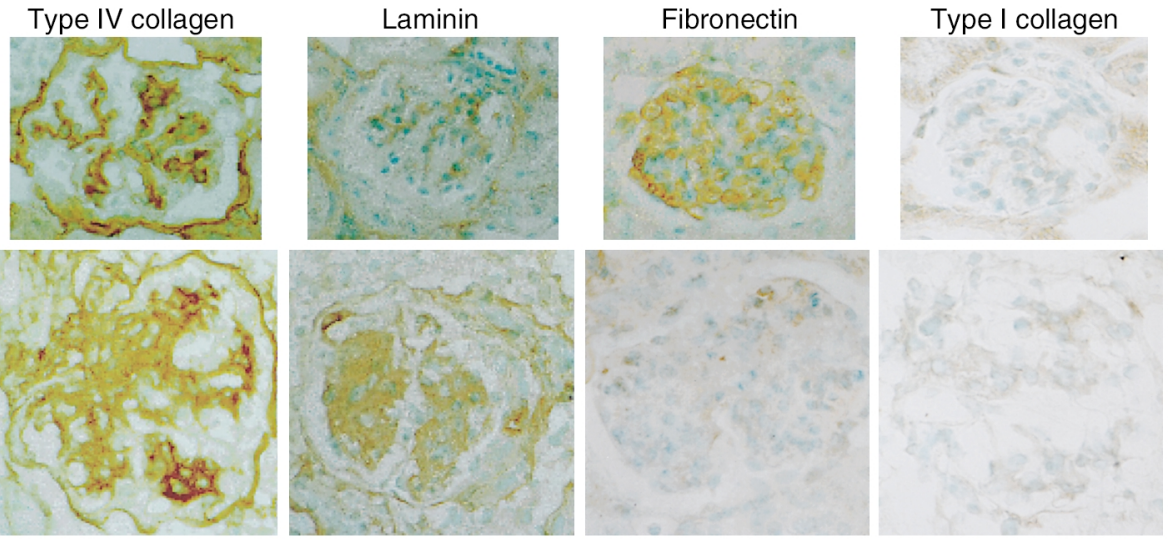

\section{Figure 6}

Immunohistochemical analysis of ECM. Marked deposition of type IV collagen and laminin was observed in the glomeruli of transgenic mice, while the amount of fibronectin was much less in the transgenic animals than in wild-type mice. Accumulation of type I collagen was not observed in either wild-type or transgenic animals. Upper row, a wild-type mouse; lower row, a megsin transgenic mouse. $\times 200$.

manner thereafter. Of note, immune complex deposition was markedly augmented in glomeruli of the transgenic mice when renal changes developed at 40 weeks (Figure 7).

Electron microscopic analysis confirmed these findings. While we observed a small number of electrondense deposits in the mesangial area of some nontransgenic mice (Figure 8), the presence of electron-dense deposits was markedly augmented in the transgenic mice; sizes of the deposits varied $(100-1000 \mathrm{~nm})$. Hypercellularity in the mesangial area of the transgenic mice was due to an increase in the number of mesangial cells, accompanied by an expansion of matrix. Pathological findings were confined to the mesangial area. Capillary endothelium generally maintained a normal appearance, and endothelial fenestrations remained preserved. Podocytes also remained intact, maintaining normal morphologic features of foot processes. Cortical tubules demonstrated normal appearances. Infiltration of mononuclear or polymorphonuclear leukocytes was undetectable.

An augmented immune complex deposition was already observed in transgenic mice at 20 weeks both by immunofluorescence studies and electron microscopic analysis. At 6 weeks, we observed no immune complex deposition in control animals or transgenic mice.

Mesangial expansion in anti-GBM nephritis persisted only in megsin transgenic mice. In order to examine whether the stressed mice overexpressing megsin would develop an overt glomerulopathy, we induced anti-GBM nephritis in the transgenic and wild-type mice and evaluated the degree of matrix expansion (Figure 9). Animals from both groups developed the same degree of mesangial matrix expansion at day 7 . At day 28 , mesangial matrix expansion persisted in transgenic mice, whereas that in wild-type animals improved. Our semiquantitative analysis showed that glomerular deposition of mouse IgG was essentially the same between the two groups at day 28. Serum creatinine levels of transgenic mice and wild-type animals did not differ throughout the time course $(0.11 \pm 0.01$ vs. $0.10 \pm 0.02 \mathrm{mg} / \mathrm{dl}$, respectively, at day 7 , and $0.09 \pm 0.03$ vs. $0.08 \pm 0.04 \mathrm{mg} / \mathrm{dl}$, respectively, at day 28).
Megsin inbibits the plasmin activity in vitro. Since the P17-P8 sequence of megsin (EGTEATAAT) was consistent with the consensus sequence among inhibitory serpins (EGTEAAAAT) (1), we evaluated the activity of megsin on serine proteases by in vitro binding studies. SDS-PAGE revealed appearance of a new band when recombinant megsin was mixed with plasmin, but not other serine proteases (t-PA and thrombin in Figure 10a). The binding of megsin to plasmin was also confirmed by SELDI-TOF mass spectrometry: in a reaction mixture of megsin and plasmin, a peak was identified with a molecular mass of $120,163 \mathrm{Da}$, which was consistent with the value of megsin $(46,883 \mathrm{Da})$ and plasmin $(73,264 \mathrm{Da})$ complexes. The complex formation was not observed when recombinant megsin was heat-inactivated. All these studies suggested that megsin bound to plasmin.

In addition, functional assays demonstrated an inhibitory effect of megsin on the enzymatic activity of plasmin (Figure 10b). By contrast, neither ovalbumin nor albumin inhibited the plasmin activity, demonstrating the specific activity of megsin. Inactivated megsin did not exhibit such an activity. The inhibitory activity of megsin was also confirmed using another substrate of plasmin (Boc-Val-Leu-Lys-MCA) (data not shown).

\section{Discussion}

Our data demonstrate that megsin has a biologically relevant influence on mesangial cell functions. Indeed, overexpression of this protein results in progressive
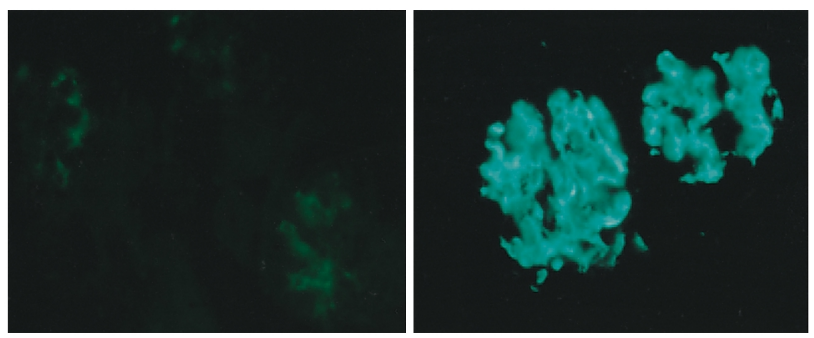

\section{Figure 7}

Immunofluorescent studies of Ig's. Diffuse granular deposits of IgC and complement were augmented in the mesangial area of 40-weekold $F_{1}$ transgenic mice (line $A$ ) (right) as compared with wild-type mice of the same age (left). $\times 50$. 


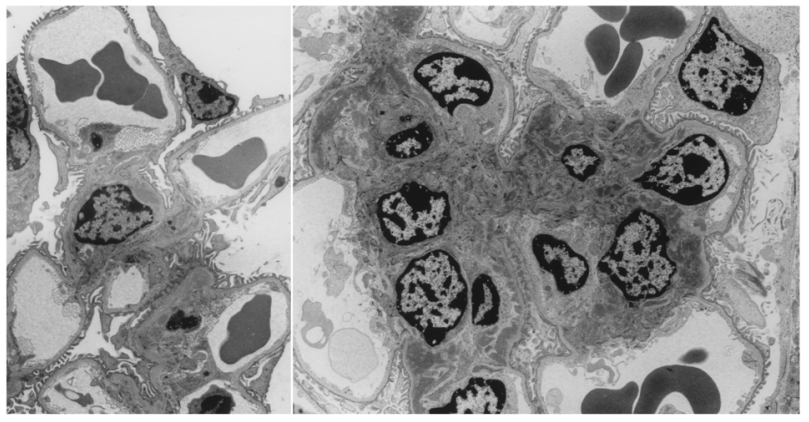

Figure 8

Electron microscopic analysis. Severe matrix expansion with numerous electron-dense deposits was detected in the mesangial matrix of $F_{1}$ megsin transgenic mouse (line A) (right). Glomerular endothelial cells and epithelial cells remained intact. Left, a wild-type mouse. $\times 2500$.

mesangial matrix expansion and in an increase in the number of mesangial cells. These pathological changes were observed in two independent lines of megsin transgenic mice. The disorders are thus specific for megsin overexpression.

The pathomechanism of the glomerular disease observed in megsin transgenic mice still remains unknown. Mesangial ECM mass is constantly regulated by its synthesis and degradation (9-13), the latter being determined by the balance existing between proteinases and their inhibitors. Plasminogen activator inhibitor-1 (PAI-1), a member of the serpin superfamily and the major physiological inhibitor of plasminogen activators, has important effects on glomerular matrix degradation (14-16). Recent studies demonstrated reduction of degradation of GBM in experimental anti-GBM nephritis by two other serine proteinase inhibitors, $\varepsilon$-aminocaproic acid and aprotinin (17). If proteinase inhibitors predominate, increased matrix accumulation and glomerulosclerosis will ensue. Although we cannot exclude a possibility that an increased number of the glomerular cells led to accumulation of more mesangial matrix, it appears more likely that overexpression of megsin, a novel member of the serpin superfamily, tipped the balance toward lower matrix degradation and an expanded matrix volume.

To further investigate a role of megsin in vivo, we employed the anti-GBM nephritis model in mice. The animal model of anti-GBM nephritis shares major pathogenic features with the human equivalent and is often used as a representative model of proliferative glomerulonephritis in mice (8). Our experiments demonstrated that megsin transgenic mice of antiGBM nephritis developed persistent mesangial matrix expansion, supporting a possible role of megsin as a functional serpin in regulating matrix deposition. The reason(s) for an increased number of mesangial cells in megsin transgenic mice may be explained by such alterations of the microenvironment of mesangial matrix, which plays an important role in mesangial cell proliferation and differentiation (18).
Immune complexes were present in increased numbers together with Ig's and complement in the expanded mesangial matrix in megsin transgenic mice. While previous glomerulonephritis models demonstrated immune complex deposition in the mesangial area, the pathogenesis of immune complex deposition in these models was exclusively ascribed to augmented production of immune complexes. Although we observed essentially the same pathological phenomenon, it should be noted that we induced the disease by genetically manipulating mesangial function. It is unlikely that augmented production was a primary mechanism of immune complex deposition in megsin transgenic mice, because the levels of circulating immune complexes in transgenic mice were at best trivial. The explanation of this phenomenon should be found in a decreased capacity of mesangial cells to clear macromolecules $(19,20)$; mesangial cells are indeed equipped with $\mathrm{Fc}$ receptors for the uptake of immune complexes $(21,22)$. We suggest that overexpression of megsin, a mesangium-predominant gene, may lead to mesangial dysfunction, impair the degradation and disposal of
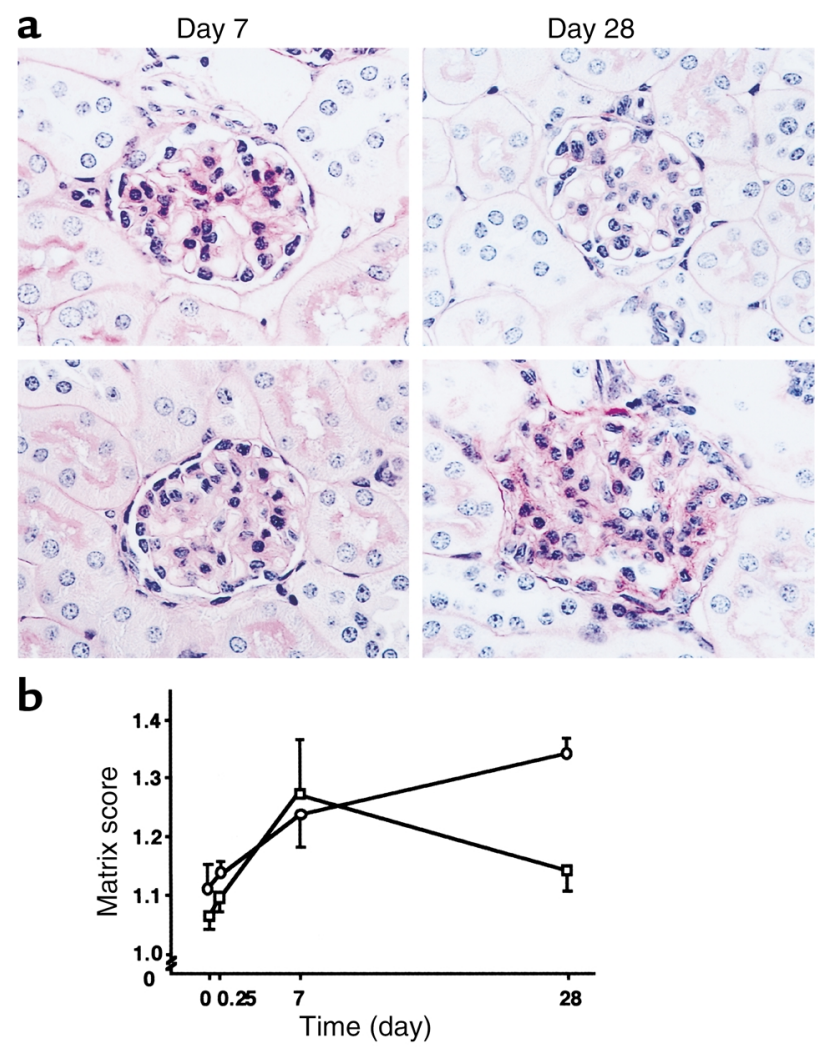

\section{Figure 9}

Anti-GBM nephritis experiments. (a) PAS staining of anti-GBM nephritis mouse kidneys. While glomerular injury in transgenic mice and wild-type mice was essentially the same at day 7 , only wild-type mice showed significant improvement at day 28. Upper panels, a wild-type mouse; lower panels, a megsin transgenic mouse. $\times 200$. (b) Semiquantitative analysis of mesangial matrix expansion of antiGBM nephritis mice. Note that mesangial matrix expansion persisted at day 28 in the transgenic mice, whereas it improved in wild-type mice. Squares, wild-type mice; circles, megsin transgenic mice. 
b

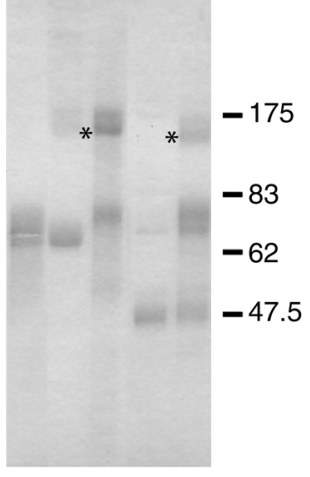

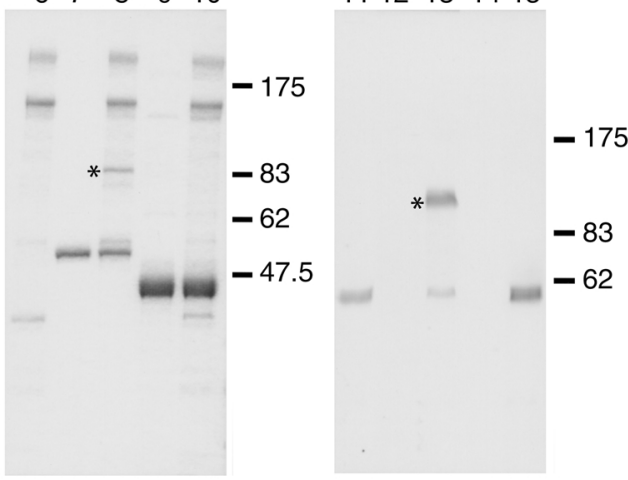

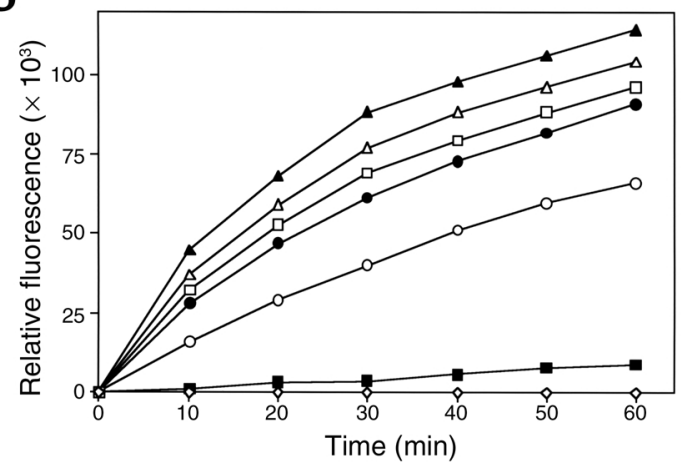

\section{Figure 10}

Functional assays of megsin. (a) SDS-PAGE under a nonreduced condition, followed by CBB staining (left and middle panels) or immunoblotting using anti-t-PA antibody (right panel), revealed functional binding of megsin to plasmin, but not to other serine proteases such as $t$-PA and thrombin. Lane 1, plasmin; lane 2, antiplasmin; lane 3, plasmin plus antiplasmin; lane 4, megsin; lane 5, plasmin plus megsin; lane 6, thrombin; lane 7, antithrombin; lane 8, thrombin plus antithrombin; lane 9, megsin; lane 10, thrombin plus megsin; lane 11, t-PA; lane 12, PAI-1; lane 13, t-PA plus PAI-1; lane 14, megsin; lane 15, t-PA plus megsin. The complex of serine protease and serpin is indicated by asterisks. (b) The inhibitory effect of megsin to plasmin activity. Diamonds, substrate alone; open squares, plasmin; filled squares, plasmin and antiplasmin (1:2); open circles, plasmin and megsin (1:10); filled circles, plasmin and inactivated megsin (1:10); open triangles, ovalbumin; filled triangles, albumin.

immune complexes, and alter the microenvironment of mesangial matrix. However, it should be noted that we did not observe any difference in mouse IgG deposition between anti-GBM nephritis transgenic mice and wildtype mice (12 weeks old) at day 28 . Although a limited sensitivity of our semiquantitative analysis may be the reason that we could not detect a difference, matrix expansion of these anti-GBM nephritis transgenic mice could be attributed to imbalance of matrix degradation rather than a decreased capacity of mesangial cells to clear macromolecules.

In order to identify potential substrates of megsin, we performed assays using recombinant megsin. Our binding and functional studies suggested that megsin binds to plasmin and has an inhibitory effect on the enzymatic activity of plasmin. In the context of mesangium matrix turnover, the plasminogen activator/plasmin cascade plays an important role through its ability to directly degrade some matrix components (23). Furthermore, plasmin also induces activation of matrix metalloproteinase, resulting in indirect degradation of extracellular matrix (24). The amount of plasmin generation is tightly regulated through the inhibition by specific inhibitors that belong to the serpin family, such as PAI-1, PAI-2, and PAI-3 (25-27). Our results, together with these reports, suggest that megsin may act as an inhibitor of plasmin and related serine proteases, leading to alterations of mesangium matrix turnover. While megsin may have some other biological substrates in glomeruli, our findings clearly demonstrate that megsin serves as a functional serpin. Although megsin is normally confined to the glomerular mesangium, the transgenic model reported in this paper is characterized by the expression of megsin in all tissues. This feature is due to the ubiquitous promoter for the transgene. Although immunohistochemical studies revealed the presence of megsin in a host of tissues as well as in nonmesangial areas of the kidney, pathogenic effects of megsin overexpression were restricted within glomeruli. This finding may suggest specific localization of megsin ligand (serine protease) other than plasmin within the mesangium. In other words, a role of megsin appears restricted to the mesangium for two reasons: first, because it is normally expressed only there, but also, second, because it requires the existence of unknown megsin ligand present only in this area.

Do our observations have any relevance for the understanding of human pathology? The described lesions are different from those observed in the aging kidney: the latter is characterized by a mild expansion of the mesangial matrix, resulting in glomerular obsolescence without an increased number of mesangial cells (28-30). By contrast, the coexistence of mesangial proliferation, matrix expansion, and increased immune complex deposition is frequently observed in human glomerular diseases. However, when they are present in a renal biopsy, the pathologist cannot unravel the sequence of pathological events. Our transgenic mice provide a unique opportunity to follow sequential events in a disease model relevant to human kidney diseases.

In conclusion, megsin transgenic mice developed progressive mesangial matrix expansion, which was associated with an increase in the number of mesangial cells. These glomerular lesions were accompanied by an augmented immune complex deposition, demonstrating a biological role of megsin as a functional serpin. 


\section{Acknowledgments}

We thank C. van Yepersele de Strihou for helpful discussions and T. Watanabe, K. Isaka, M. Asakura, T. Umezono, and $\mathrm{H}$. Uemura for their excellent technical assistance. This study was supported by grants from the Japan Science and Technology Cooperation (to T. Miyata), from the Japanese Ministry of Science and Technology Agency (Millenium Project to T. Miyata), from the Japanese Ministry of Health, Labour and Welfare (to T. Miyata), from the Japanese Ministry of Education, Culture, Sports, Science and Technology (to R. Inagi and K. Kurokawa), from Sankyo Foundation of Life Science (to R. Inagi), and from the Takeda Science Foundation (to R. Inagi).

1. Miyata, T., et al. 1998. A mesangium-predominant gene, megsin, is a new serpin up-regulated in IgA nephropathy. J. Clin. Invest. 102:828-836.

2. Suzuki, D., et al. 1999. Expression of megsin mRNA, a novel mesangiumpredominant gene, in the renal tissues of various glomerular diseases. $J$. Am. Soc. Nephrol. 10:2606-2613.

3. Inagi, R., et al. 2001. Specific tissue distribution of megsin, a novel serpin, in the glomerulus and its up-regulation in IgA nephropathy. Biochem. Biophys. Res. Commun. 286:1098-1106.

4. Nangaku, M., et al. 2001. Cloning of rodent megsin revealed its up-regulation in mesangioproliferative nephritis. Kidney Int. 60:641-652.

5. Kawarabayashi, T., et al. 1996. Accumulation of $\beta$-amyloid fibrils in pancreas of transgenic mice. Neurobiol. Aging. 17:215-222.

6. Hogan, B., Costantini, F., and Lacy, E. 1986. Manipulating the mouse embryo: a laboratory manual. Cold Spring Harbor Laboratory Press. Cold Spring Harbor, New York, USA. 487 pp.

7. Sekine, H., et al. 2001. Complement component C3 is not required for full expression of immune complex glomerulonephritis in MRL/lpr mice. J. Immunol. 166:6444-6451.

8. Hisada, Y., et al. 1999. Angiotensin II plays a pathogenic role in immunemediated renal injury in mice. J. Clin. Invest. 103:627-635.

9. Johnson, R.J., Lovett, D., Lehrer, R.I., Couser, W.G., and Klebanoff, S.J. 1994. Role of oxidants and proteases in glomerular injury. Kidney Int. 45:352-359.

10. Davie, M., Martin, J., Thomas, G.J., and Lovett, D.H. 1992. Proteinases and glomerular matrix turnover. Kidney Int. 41:671-678.

11. Baricos, W.H., and Shah, S.V. 1991. Proteolytic enzymes as mediators of glomerular injury. Kidney Int. 40:161-173.

12. McLennan, S., Fisher, E., Yue, D.K., and Turtle, J.R. 1994. High glucose concentration causes a decrease in mesangium degradation. A factor in the pathogenesis of diabetic nephropathy. Diabetes. 43:1041-1045.
13. Davies, M., Coles, G.A., Thomas, G.J., Martin, J., and Lovett, D.H. 1990. Proteinases and the glomerulus: their role in glomerular diseases. Klin. Wochenschr. 68:1145-1149.

14. Fogo, A.B. 2000. The role of angiotensin II and plasminogen activator inhibitor-1 in progressive glomerulosclerosis. Am. J. Kidney Dis. 35:179-188.

15. Loskutoff, D.J. 1993. A slice of PAI. J. Clin. Invest. 92:2563.

16. Nakamura, S., Nakamura, I., Ma, L., Vaughan, D.E., and Fogo, A.B. 2000. Plasminogen activator inhibitor-1 expression is regulated by the angiotensin type 1 receptor in vivo. Kidney Int. 58:251-259.

17. Hruby, Z., et al. 2000. Mechanism of antinephritic effect of proteinase inhibitors in experimental anti-GBM glomerulopathy. Res. Exp. Med. (Berl.) 199:295-307.

18. Turck, J., Pollock, A.S., Lee, L.K., Marti, H.P., and Lovett, D.H. 1996. Matrix metalloproteinase 2 (gelatinase A) regulates glomerular mesangial cell proliferation and differentiation. J. Biol. Chem. 271:15074-15083.

19. Farquhar, M.G., and Palade, G.E. 1961. Glomerular permeability. II. Ferritin transfer across the glomerular capillary wall in nephrotic rats. J. Exp. Med. 144:699-715.

20. Sterzel, R.B., and Rupprecht, H.D. 1997. Glomerular mesangial cells. In Immunologic renal diseases. E.G. Neilson and W.G. Couser, editors. Lippincott-Raven Publishers. Philadelphia, Pennsylvania, USA. 595-626.

21. Neuwirth, R., et al. 1988. Evidence for immunoglobulin Fc receptormediated prostaglandin 2 and platelet-activating factor formation by cultured rat mesangial cells. J. Clin. Invest. 82:936-944.

22. Santiago, A., Mori, T., Satriano, J., and Schlondorff, D. 1991. Regulation of Fc receptors for IgG on cultured rat mesangial cells. Kidney Int. 39:87-94.

23. Mclennan, S.V., et al. 2000. Effects of glucose on matrix metalloproteinase and plasmin activities in mesangial cells: possible role in diabetic nephropathy. Kidney Int. Suppl. 77:S81-S87.

24. Baricos, W.H., Cortez, S.L., el-Dahr, S.S., and Schnaper, H.W. 1995. ECM degradation by cultured human mesangial cells is mediated by a PA/plasmin/MMP-2 cascade. Kidney Int. 47:1039-1047.

25. Rerolle, J.P., Hertig, A., Nguyen, G., Sraer, J.D., and Rondeau, E.P. 2000. Plasminogen activator inhibitor type 1 is a potential target in renal fibrogenesis. Kidney Int. 58:1841-1850.

26. Eitzman, D.T., and Ginsburg, D. 1997. Of mice and men. The function of plasminogen activator inhibitors (PAIs) in vivo. Adv. Exp. Med. Biol. 425:131-141.

27. Scott, F.L., et al. 1999. Human ovalbumin serpin evolution: phylogenic analysis, gene organization, and identification of new PI8-related genes suggest that two interchromosomal and several intrachromosomal duplications generated the gene clusters at 18q21-q23 and 6p25. Genomics. 62:490-499.

28. Baylis, C., and Corman, B. 1998. The aging kidney: insights from experimental studies. J. Am. Soc. Nephrol. 9:699-709.

29. Couser, W.G., and Stilmant, N.M. 1975. Mesangial lesions and focal glomerulosclerosis in the aging rat. Lab. Invest. 33:491-501.

30. Goldstein, R.S., Tarloff, J.B., and Hook, J.B. 1988. Age-related nephropathy in laboratory rats. FASEB J. 2:2241-2251. 